\title{
Emergency Alimentary-tract Surgery in the Newborn*
}

\author{
P. P. RICKHAM, $†$ M.S., F.R.C.S., D.C.H.
}

Brit. med. F., 1966, 1, 78-79

In the following brief review it is not proposed to give detailed consideration to the many technical difficulties associated with this highly specialized branch of surgery, but to concentrate on those problems which may interest the physician suddenly called upon to deal with such an infant and to present some data which may be of use to the family doctor when he has to advise the parents of a baby suffering from alimentary-tract obstruction.

\section{Incidence}

With the exception of malformations of the central nervous system requiring urgent treatment, alimentary-tract surgery is by far the commonest surgical emergency in the neonatal period. During the first twelve years of the existence of the Liverpool Regional Neonatal Surgical Centre 2,000 newborn infants suffering from urgent surgical conditions were admitted; 179 of them had oesophageal atresia and 447 intestinal obstruction (Table I). Virtually all newborn infants with surgical conditions born in an area with a population of approximately $3,000,000$ and an annual birth rate of 60,000 are admitted to this unit ; the incidence of children admitted with alimentary-tract malformation in our area is therefore about one per 1,000 live births.

TABLE I.-Analysis of 2,000 Cases Admitted to the Liverpool Neonatal Surgical Unit (Main Groups of Cases Only)

\begin{tabular}{lrr|llll} 
Tumours .. &. &. & 30 & Rectal malformations & & 152 \\
Thoracic malformations &. & 75 & Oesophageal atresia & $\ldots$ & 179 \\
Genito-urinary malformations & 87 & Ontestinal obstruction &. & 447 \\
Exomphalos &.. &.. & 106 & C.N.S. malformations & $\ldots$ & 570
\end{tabular}

\section{Results}

To-day the results of alimentary-tract surgery in the newborn depend to a large extent on two factors-the infant's birth weight and the coexistence of other severe congenital malformations. The survival rate of full-term infants suffering from uncomplicated atresia of the alimentary tract should now be at least $80 \%$, but in premature infants or those suffering from other severe malformations the mortality rises steeply. This is clearly shown by our results for such conditions as intestinal atresia (Table II). By early diagnosis and treatment we tend to avoid such complications as pneumonia in oesophageal atresia or perforation, gangrene,

TABLE II.-Results of Operations for Intestinal Atresia in Absence of

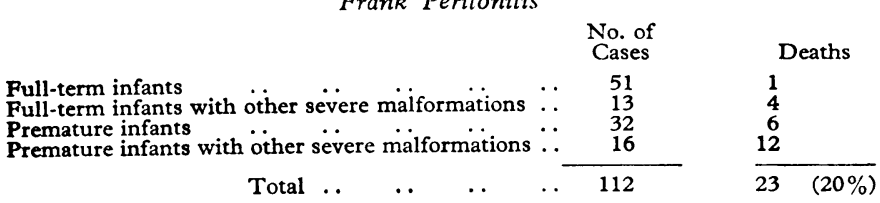

TABLE III.-Results of Operations for Intestinal Obstruction Not Needing Intestinal Resection

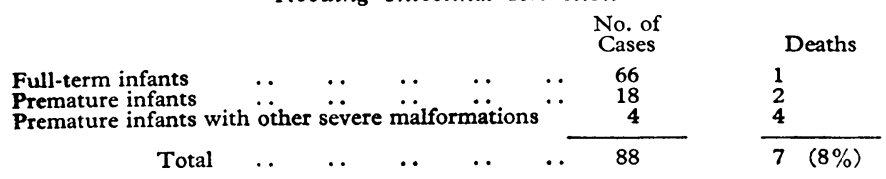

and peritonitis in infants with intestinal obstruction. When we compare the results of uncomplicated intestinal atresia (Table II), or of laparotomy for intestinal obstruction which can be relieved without intestinal resection (Table III), with the results of operation for frank meconium or bacterial peritonitis (Table IV) the difference in the operative mortality in early and late cases respectively becomes apparent.

TABLE IV.-Results of Operation for Frank Bacterial or Meconium Peritonitis

\begin{tabular}{|c|c|c|}
\hline 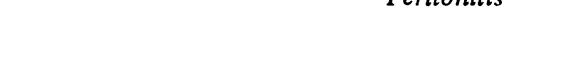 & $\begin{array}{l}\text { No. of } \\
\text { Cases }\end{array}$ & Deaths \\
\hline $\begin{array}{l}\text { Full-term infants } \\
\text { Full-term infants with other severe malformations } \\
\text { Premature infants } \\
\text { Premature infants with other severe malformations }\end{array}$ & $\begin{array}{r}32 \\
3 \\
13 \\
5\end{array}$ & $\begin{array}{l}7 \\
3 \\
8 \\
5\end{array}$ \\
\hline Total & 53 & $(43 \%)$ \\
\hline
\end{tabular}

\section{Early Diagnosis and Initial Treatment in Cases of Obstruction of the Alimentary Tract}

The presenting signs and symptoms as well as the initial treatment depend on whether the obstruction is in the oesophagus or in the intestine.

\section{Oesophageal Obstruction}

The presence of hydramnios is very suggestive of alimentary-tract obstruction, especially oesophageal atresia. Prematurity is so often associated with this condition that special care should be taken when examining premature infants. An absent anus is frequently associated with oesophageal atresia, and this association must always be borne in mind. The presenting signs, such as the constant accumulation of saliva and mucus in the mouth and the dribbling of saliva, are still occasionally missed, with the unfortunate result that feeding is commenced. So long as it has been made a rule that the first feed should never be milk, but water, the worst dangers of aspiration pneumonia may be avoided, but even a water feed may drown a premature infant suffering from oesophageal atresia. Considerable evidence has accumulated that the worst type of pneumonia complicating oesophageal atresia is produced by regurgitation of stomach juice through the tracheo-oesophageal fistula into the bronchial tree. Vomiting is particularly apt to occur if the infant suffers from any sort of distress, and this makes feeding by mouth very dangerous. Attacks of respiratory distress and cyanosis may occur without feeding, especially when the infant's position is changed, but cyanosis and dyspnoea are less common than the bringing up of mucus and saliva.

Once the practitioner's suspicion has been alerted the diagnosis should be confirmed by passing a rubber catheter through the mouth down the oesophagus. In a full-term infant with atresia the catheter will become arrested at a distance of about $4 \mathrm{in}$. $(10 \mathrm{~cm}$.) from the lips, but if the catheter is soft and the blind proximal oesophageal pouch dilated it is occasionally possible for a length of catheter to coil up in the

\footnotetext{
- Paper read at the Annual Clinical Meeting of the British Medical Association, Dundee, 1965.

† Consultant Paediauric Surgeon, Alder Hey Children's Hospital, Liverpool.
} 
oesophageal pouch. Difficulties in swallowing and a respiratory distress on feeding also occur in such conditions as intracranial birth injuries, local malformations of the pharynx, diaphragmatic hernia, and tracheo-oesophageal fistula without atresia. In all these cases a catheter can be passed into the stomach.

\section{Intestinal Obstruction}

The incidence of prematurity is not as high with intestinal obstruction as it is with oesophageal malformations, nor is the incidence of hydramnios. Of the associated malformations mongolism, commonly seen in children suffering from duodenal atresia, should be mentioned.

Vomiting is always the presenting symptom, and as the obstruction is usually below the level of the ampulla of Vater the vomitus is nearly always bile-stained. Vomiting occurs early when the obstruction is high up in the intestinal tract, as for instance in the duodenum. It will then be profuse and may be projectile in character. It is late in onset and neither profuse nor forceful in low-intestinal obstruction. There are few other causes of vomiting of green fluid in the newborn, and as a rule they can be easily differentiated. Intracranial birth injuries are sometimes associated with bile-stained vomiting. Swallowing of meconium-stained liquor may produce meconium gastritis and vomiting of greenish fluid; and some small premature infants may vomit bile in the absence of obstruction. The dangers of vomiting in infancy (such as aspiration of vomitus), dehydration, and ion depletion are so well known that they need not be stressed.

Abdominal distension is another presenting symptom. It is minimal in upper-intestinal obstruction but massive in obstruction of the distal part of the intestine. Newborn infants, with their relatively weak abdominal musculature, can have severe distension with a tensely protuberant abdomen, shiny abdominal skin, and dilated veins traversing the abdominal surface. Bearing in mind the relatively underdeveloped state of the newborn infant's lungs and the fact that respiration is almost entirely diaphragmatic, the danger that severe abdominal distension may interfere with efficient respiration becomes obvious.

Constipation is not an important symptom. In incomplete obstruction, meconium and, later on, milk stools can be passed; but even in complete obstruction one or two small meconium stools are often observed. The passing of a plug of greyish meconium may be indicative of complete highintestinal obstruction. If it is followed by the passing of fluid meconium and flatus-the so-called meconium plug syndrome - it has been thought to be of only temporary clinical significance, but in our experience many of these children will obstruct again later, and will finally turn out to be cases of Hirschsprung's disease.

When the diagnosis of intestinal obstruction is suspected, treatment must be immediately directed towards the prevention of further vomiting and the relief of abdominal distension. This is best carried out by passing a soft rubber or plastic catheter through the nostril into the stomach, strapping it to the nostril, and deflating the gastro-intestinal tract by continuous and/or repeated stomach aspiration. In contrast with older children and adults, the infant's gastro-intestinal tract can be completely deflated even in the presunce of lowintestinal obstruction.

\section{Organisation of a Neonatal Surgical Service}

Unless treatment of these relatively uncommon cases is concentrated in a few widely separated centres, surgeons, anaesthetists, and nurses will not get sufficient experience to obtain consistently good results. Modern neonatal surgery requires a team of highly specialized medical and nursing personnel as well as a considerable amount of special equipment; and because of this, and for financial reasons, it is imperative to concentrate this work.

The setting up of neonatal surgical centres will be of little avail unless early diagnosis, initial management, and transport of these infants to the Centre are efficiently organized. This necessitates close contact between the surgeons working at the Centre and the obstetricians, paediatricians, and midwives working in the maternity departments as well as the practitioners and midwives delivering babies at home.

\section{Transport}

The establishment of widely dispersed neonatal surgical centres necessitates transport of these infants over considerable distances. This can be quite a formidable problem even in a highly industrialized area like our own. The infants must be accompanied by a qualified nurse experienced in dealing with this emergency. Oesophageal and intestinal cases must be nursed lying on their side to prevent aspiration of mucus and vomitus. They must be well protected against heat loss by transporting them in padded carry-cots, well wrapped in blankets, and in the cold season by hot-water bottles placed outside the blankets. The nurse must always have a mucusextractor handy, ready at any moment to clear the air passages by sucking out the mucus or vomitus. Although oxygen is usually provided, administration by the tube-and-funnel method is rarely of much value. Patients with intestinal obstruction are transported with a stomach catheter in situ, and the nurse is instructed to aspirate the stomach, with the aid of a syringe, at ten-minute intervals.

Premature infants should be transported in portable incubators at a constant temperature of at least $85^{\circ} \mathrm{F} .\left(29.4^{\circ}\right.$ C.) and an atmospheric humidity approaching $100 \%$.

Provided these precautions are taken, the infants travel well even over long distances; but with the increasing congestion on our roads and consequent delay in transport careful consideration must be given to the possibilities of transportation by helicopter in the future.

\section{Summary}

Alimentary-tract surgery is the second commonest emergency in the neonatal period. Of 2,000 newborn infants suffering from urgent surgical conditions admitted to the Liverpool Regional Neonatal Surgical Centre 626 had obstruction of the alimentary tract.

The organization of a neonatal surgical service dealing with these malformations, and the early diagnosis and initial treatment of emergency cases of alimentary-tract obstruction, are discussed. Early diagnosis and efficient pre-operative management play a very important part in lowering the mortality associated with these conditions. 